\title{
THE CLINICAL VARIETIES OF DISORDER OF FUNCTION.(1)
}

\author{
By A. E. CLARK-KENNEDY, M.D., F.R.C.P. \\ Honorary Assistant Physician to the London Hospital and Consulting Physician to the \\ King Edward Memorial Hospital, Ealing.
}

I imagine that many if not most of you here to-day must have passed in your student days through this experience. When you were clinical clerks in medical wards you frequently saw patients admitted to the hospital with diagnoses such as gastric ulcer and carcinoma of the stomach in which subsequent investigation by X-rays and other methods failed to substantiate the diagnosis of organic disease with which they had been admitted. But such patients had symptoms, and therefore, in the ordinary sense of the word, were ill. If you enquired what was the matter with them, you discovered that the house physician had no clear ideas: you were probably told that they were "functional" cases. Anyhow, their symptoms cleared up quickly under the ordinary hospital regime. As, therefore, they did not appear to require any particular form of treatment, or appear to be of any particular interest to anybody, they were sent back to the out-patient clinic or referred to their doctors without any particular diagnosis except a statement that no evidence of organic disease had been discovered, without any explanation to the patient of the nature and origin of his or her symptoms, and without any particular form of treatment having been prescribed.

But it is these "functional" cases that form a large proportion of patients in general practice and swell the army of chronic cases which attend the out-patient clinic of any general hospital. It is to these "functional" cases, therefore, that $\mathrm{P}$ propose to pay particular attention in these lectures, because while being numerous they are little understood, and being regarded as of little interest they are seldom taught about; and as these cases present digestive symptoms more frequently than any others, I have chosen "indigestion" as the subject of my first few lectures.

The term "functional disease" has no significance. All disease is functional in that a person does not complain of symptoms until one or another function of the body or element of the mind becomes disturbed. Organic disease obviously causes disturbance of function, but the term "functional disease" is usually understood to mean or imply the absence of any organic structural cause for the patient's symptoms. There are, however, several ways in which the function of some organ of the body may be upset apart from a structural disorder, and a simple division of ill people into those that are "organic" and those that are "functional" does not satisfy the requirement of every-day medical practice. As we must have some more adequate working classification of disease, I propose to consider to-day, with special reference to the digestive system, the different ways in which the normal working of the human body can be upset, so that symptoms are produced, and the patient is driven to consult his doctor. This is not an academic question, but one the proper understanding of which is of fundamental importance in the practice of medicine.

In the first place, disorder of function with the production of symptoms may occur in an individual quite apart from organic disease as usually understood or neurosis as usually defined. Physiological defect or weakness may be inborn and

(1)Being the first of a course of lecture-demonstrations on general medicine from the point of view of common symptoms being given on behalf of the Fellowship of Medicine and the Post-Graduate Medical Association during 1934, on Tuesdays, at 2.30 p.m., the Medical Society of London, 11, Chandos Street. 
present from the start, and may be hereditary or familial ; or it may. appear as a failure of proper post-natal physiological development. On the other hand, it may be acquired as the result of bad habits, accident, or the stress of environment. Congenital pyloric stenosis and Hirschsprung's disease for instance are examples of disordered physiological function present at or developing soon after birth. Then there are also the rare inborn disorders of metabolism, hereditary diseases such as hæmophilia, Friedreich's ataxia, and familial acholuric jaundice, and the family predisposition to certain diseases such as peptic ulcer, high blood pressure, pulmonary tuberculosis, and some allergic conditions. Failure of postnatal physiological development accounts for the child who gets diabetes at the age of ten or fails to develop sexually at puberty on account of deficiency of some ductless gland. Exhaustion of physiological function with advancing age would appear to be the cause of myxœedema, pernicious anæmia, and some cases of diabetes. Physiological accident may account for some cases of acquired allergy such as hay fever and asthma, while the environment or social conditions and customs must be blamed for conditions such as rickets, scurvy, beri-beri and osteomalacia.

It is not to these text-book "diseases", however, that I wish to direct your attention, but to the more common and less spectacular disorders of physiological function to which the gastro-intestinal tract is particularly prone. Just as people all differ to some extent in structure, so the different systems of their bodies differ in efficiency. One man has greater muscular development than another. One athlete has better cardio-vascular development and can run further or faster than his rival. One person is blessed with a better digestion than another in whom the limited digestive capacity of the child has failed to develop into that of the normal adult. One man has bowels which act regularly: another has been constipated from infancy, or may have become constipated as the result of failing to develop the necessary habit. Trained as most of us have been in the strict school of organic disease, or prejudiced in favour of the psychological approach to medicine as some have become, we are sometimes too ready to suspect organic disease when no organic disease is present, or too apt to postulate some psychological basis for the patient's symptoms without adequate reason. Give each of two normal people one half of the same green apple to eat; and one may get indigestion in consequence and the other not, but it would be as absurd to say that this unfortunate dyspeptic was suffering from organic disease, as it would be libellous to condemn him as a neurotic. We must admit, therefore, that many people suffer from a poor physiology in some particular respect, and are handicapped in life by some organ inferiority of which they are usually aware. The symptoms produced may be trivial in themselves, and there is little evidence that disorder of function of this kind predisposes to organic disease, but these symptoms, trivial as they may be, if mishandled or self-treated, are likely to start a train of fears and anxieties in the mind of a neurotic person.

In the second place, disorder of bodily function may occur with consequent production of symptoms when a person is emotionally upset. The heart, blood vessels, sweat glands, the whole gastro-intestinal tract, and all the glands associated with digestion, are under the control of the autonomic nervous system, and the sympathetic division of this system is activated by the emotions of fear, anxiety, and fright. Under the stimulus of fear, the heart beats faster, digestion ceases, peristalsis stops, and the blood is deflected from the splanchnic area into the skeletal muscles. These changes were intended by nature to prepare the body for vigorous action. Under the conditions of modern civilised life, however, this 
arrangement serves no useful purpose, for we no longer fight or run away. There are no such simple methods of escape from situations which make us anxious or afraid. Tachycardia, sweating and palpitation are of no physiological value in a situation from which there is no escape. Loss of appetite, indigestion and sleeplessness serve no useful purpose in sorrow, anxiety or fear. Some people are fortunate in life, or, born phlegmatic, worry little, or are so constituted that their sympathetic nervous systems leave their viscera alone. Such people digest their meals under all circumstances, and their physiology is left undisturbed by anxiety and sleepless nights. Other people are less fortunate and worry too much over little things, or are so constituted that their sympathetic nervous systems will not leave their physiology alone. In such people a meal eaten under mental stress is ill digested, and bad news at breakfast time may interfere with the usual action of the bowels.

Many patients who complain of symptoms are not suffering from some primary disorder of physiology or from organic disease, but from chronic fear, anxiety or mental unrest. They may be over-anxious about their own health, or anxious, not necessarily without reason, about a wife or child. They may have lost confidence in their ability to do their work, or be apprehensive about losing their occupation. They may be secretly terrified of getting some disease, or scared of the possible consequences of some excess committed; or their peace of mind may be disturbed by some conflict between the urge of instinct and conscience or the conventions of society. A neurotic constitution would appear to be hereditary, and a conflict may be determined by the patient's upbringing, or by the circumstances of his environment. Fear of disease may be suggested by the illness of a friend or the death of a near relative, or by an article on cancer in some lay journal or an advertisement for some patent medicine. I saw an advertisement in an American journal for some drug claimed to reduce the blood pressure headed: "Is your blood pressure rising"? Such advertisements do much harm in this age of materialism in which most people have ceased to exercise themselves about the welfare of their souls, and unfortunately worry instead about the health of their bodies, and in their search after health have lost both health and happiness. Or the suggestion of disease may come from within in which case the particular form is likely to be determined by the patient's particular physiological weakness. Thus extrasystoles, palpitation, and shortness of breath, or præcordial pain, really gastric in origin, may suggest heart disease, or frequent headaches, high blood pressure. A little pain after meals may suggest the idea of cancer of the stomach, constipation the idea of obstruction, or a little colon spasm the idea of appendicitis. Ideas of this kind arising from within are readily confirmed by suggestion from without as by the death of a friend from apoplexy, or well-meaning propaganda about the early recognition of cancer, or an advertisement for the latest aperient, or an unguarded statement by a medical practitioner about appendicitis. The patient with the idea of high blood pressure, conscious of the beating of his heart, cannot sleep. The patient with extra systoles may lie awake waiting for the next missed beat, afraid his heart will stop. Such sleepless nights impair the general health and wear down the resistance of the mind to anxious thoughts. The idea of obstruction by causing anxiety in the patient's mind through the sympathetic aggravates the constipation, and although there is little evidence that any recognised organic disease can be attributed to intestinal toxæmia, constipation makes many people depressed, and the depression thus produced aggravates the anxiety state. The idea of cancer means meals eaten under the stress of fear, and fear inhibits digestion, and aggravates the indigestion. 
Such patients naturally enough start dieting themselves, and by starving themselves of meat and other food rich in iron may develop anæmia of the hypochromic type. Not only does the mind react on the body through the autonomic nervous system, but bodily states react back upon the mind.

In the third place, disorder of function may be produced, not by anxiety, but by direct intervention of the mind. Not all patients want to be well. Some definitely would like to be ill, or to remain ill, because invalidism means more attention or provides a comfortable and respectable method of escape from an unpleasant situation. Few actually malinger, that is to say, consciously pretend to be ill, but in some persons a repressed and subconscious desire to be ill may make use of some symptom suggested from without, or by previous organic disease, to provide a method of escape from an unpleasant situation. This is the underlying mechanism of hysteria. During the war many men were placed in situations which meant a conflict between sense of duty and self respect and a natural tendency to run away. Illness or wounds alone afforded a method of escape. In some, therefore, the not unnatural wish to be ill working in the unconscious mind made use of some symptom suggested from somewhere, or by some happening, and they became in their own conscious minds ill. They were not malingering as the whole process was subconscious, but illness thus produced provided a solution of their mental conflict and method of escape with self-respect more or less maintained from a situation which their nerves were not strong enough to stand. Hysteria is less common in civil life, and the subconscious motive keeping up the symptoms is sometimes hard to discover, but a simple instance is that of a woman who becomes an invalid to get more kindness from her husband, or develops symptoms in order to attract more attention from her family by which she thinks she is neglected. Other people who feel that they are a failure in life undoubtedly get satisfaction out of being ill and becoming a centre of interest. Such patients, having achieved their object, naturally intend to remain ill, and the "belle indifference" of the hysteric, as Janet described it, contrasts strikingly with the anxious state of the ordinary neurotic. The particular symptom chosen by the patient for the purpose may merely depend upon the latest suggestion of disease to which the patient has been subjected, but may occasionally be symbolic of the patient's particular mental conflict. I once saw a patient whose hysterical attacks simulated the crucifixion. But whichever is the case, as hysterical symptoms are produced by the intervention of the mind, they usually take the form of disorders of control of skeletal muscles or disorders or peripheral sensation. Thus fits, paralyses, aphonia, aphasia, tremor, and anæsthesia are common. Hysterical indigestion can hardly occur, but vomiting, dysphagia, and retention of urine are also common. Hysterical pain appears to exist, and if referred to the abdomen may be mistaken for true indigestion. It is an intractable pain unrelated to anything particular and for which no organic cause is to be found. The ordinary analgesics and even morphia fail to bring relief. When taken into hospital the patient may sleep quite well, but continues to complain in apparent good faith of sleepless nights. When an attempt is made to explain his pain away he reacts against it; sympathised with, however, and left with his pain, he is unexpectedly content. Hysterical pain is sometimes said to be due to a reduction of the threshold of consciousness to painful stimuli from a particular part of the body. Certainly attention directed to a carious tooth or septic finger will increase the pain, and distraction of the mind elsewhere may abolish such pain almost altogether. But patients with hysterical pain sleep, and hysterical pain is something more than this very ordinary psychological phenomenon to which most of us would admit. 
Hysterical pain serves the patient's purpose, and, if analysed carefully, will usually be found to consist of "awful feelings" rather than true pain. Such patients therefore enjoy the emotion of pain without suffering from its physical consequences.

In the fourth place disorder of physiological function may be produced by organic disease, and organic disease may interfere with normal physiological processes in three ways:-by chemical or toxic action at a distance, by reflex action, or by structural change. Obvious examples of disorder of function produced by toxic or chemical action at a distance are the convulsions of tetanus and the heart failure of diphtheria. But in many conditions e.g., meningitis, it is impossible to say whether the symptoms are due to chemical action or structural change. The distinction is, however, useful and should be made when possible for it reminds us that such apparent trivial conditions as breathlessness or fatigue after an attack of influenza, or lassitude and general ill-health associated with an abscess at the root of a tooth, are organic in origin, and are not to be attributed to primary disorders of physiological function, or diagnosed as due to anxiety states as is apt to happen if we begin pushing psychological explanations of symptoms too hard. Certainly one of the most difficult diagnoses is to say whether the fatigue of which a patient complains is psychoneurotic in origin or due to some minor organic disorder, and frequent mistakes were made during the war in the differential diagnosis between neurotic patients with cardiac symptoms and cases of pulmonary tuberculosis with tachycardia and breathlessness. Then organic disease may give rise to disorder of physiological function by reflex action. Thus a duodenal ulcer may cause pyloric spasm and obstruction, an atheromatous patch by causing spasm of a cerebral artery may lead to a transient attack of hemiplegia, and a diseased coronary artery by the same mechanism to angina pain, while disease of any viscus may give rise to hyperæsthesia over the corresponding segmental area of skin. Such reflex action is essentially local, be it an axon reflex or a true reflex through the spinal cord. Whether organic disease can cause disorder of function at a greater distance by true reflex action is uncertain. The attack of asthma in a patient with nasal polypi is often attributed to such a mechanism. At one time we heard a great deal about reflex dyspepsia from chronic inflammation of the appendix and chronic infection of the gall bladder and the reflex dyspepsia theory has in the past been used to justify many appendicectomies. But the chronically inflamed appendix is falling out of fashion, and many deny its existence as a cause of dyspeptic symptoms (as opposed to recurrent appendicular colic) altogether, while the dyspeptic symptoms associated with gall bladder disease can be more reasonably attributed to associated achlorhydria or mucous catarrh of the stomach than to hypothetical reflex action. Moreover organic disease may produce disorder of physiological function by virtue of a structural change: carcinoma of the stomach and organic pyloric obstruction, cerebral hæmorrhage and established hemiplegia, coronary thrombosis and progressive cardiac failure, as opposed to the transient disorders of physiological function provoked by reflex spasm. When organic disease causes toxic states, symptoms are usually present from the beginning. Structural lesions have, however, to progress some way before symptoms are produced unless pain is caused or function transiently upset by some reflex mechanism. Often this does not occur. Thus a person with rheumatic heart disease may be completely unaware of it for years, and in carcinoma of the stomach symptoms are often absent until it is too late.

In the fifth place, and lastly, disorder of physiological function may have no real existence as it has in anxiety neurosis and hysteria, but may exist only as 
a delusion in the patient's mind. Some cancer phobias with digestive symptoms are of this nature and no amount of persuasion or reason, or even the objective evidence of X-rays, will convince such a patient that he has not cancer or cure him of his symptoms. The condition is not very common, but may be encountered unexpectedly on occasions when you think you are only dealing with an ordinary neurotic for such patients are often not obviously insane. I remember a patient who complained of violent hunger pains suggestive of duodenal ulcer. It soon became apparent, however, that she had no organic lesion, but was possessed by the idea that she was being starved although she was eating more than any ordinary person. Her subsequent disorder of behaviour and conduct made it abundantly clear that she was suffering from a psychosis rather than a neurosis.

Disorder of physiological function, therefore, with the production of symptoms may be produced (I) by a primary or acquired physiological defect; (2) by an anxiety state of the conscious mind; (3) by a motive of the sub-conscious mind; (4) by organic disease; and (5) may be of the nature of a delusion and have no real existence outside the patient's mind. The general principles underlying the differential diagnoses of these five classes of functional disorder are obvious. A primary disorder of physiological function may be recognised by the fact that symptoms occur whenever that particular physiological function is over-taxed, or in an acquired disorder of function such as asthma or hay fever by the fact that symptoms occur as an immediate consequence of a change in the external environment such as the presence of a horse or the advent of the pollen season. Primary or acquired disorders of physiological function seldom, however, remain clear cut as sooner or later they lead in most people to an anxiety state of mind. Thus in the course of time most patients with asthma become neurotic. Disorder of function produced by, or aggravated by, suggestion and anxiety neurosis runs parallel with the emotional states of the patient's mind. Many are better when their minds are distracted from their own health by plenty to do, while others develop neurotic symptoms inconveniently whenever faced with a rush of work. The most valuable thing in the differential diagnosis between anxiety neurosis and organic disease is an accurately taken history and one taken with due attention to the patient's emotional reactions. Someone else's history is almost valueless. Patients with anxiety neurosis give a history of their troubles in an emotional way, and are usually only too ready to offer explanation of their symptoms which do not follow any particular sequence, or develop in any recognised way. A completely negative physical examination will confirm a suspicion of neurosis.

Organic disease, on the other hand, tends to follow a definite recognised course and has regular habits, and therefore gives rise to typical histories such as those obtained from patients with progressive rheumatic heart disease or long standing chronic duodenal ulcer. Moreover, for some reason, patients with organic disease are not usually neurotic, and the symptoms of organic disease are not therefore related by the patient in an emotional way and there is usually little attempt at explanation. The diagnosis of organic disease may be assisted by the recognition of certain physical signs, but a negative physical examination does not rule out the possibility of organic disease when organic disease has been suspected from the history. In such cases, however, X-rays, or a chemical or serological test will usually confirm the organic diagnosis. It is impossible for administrative and economic reasons, even if it were advisable, to submit all patients with symptoms to complete investigation. But one sees patients on whom almost every 
conceivable investigation has been performed at great expense to the hospital or patient, usually the former, and with considerable discomfort to the patient, when a little more care devoted to the history would have made it obvious from the first that all investigations were doomed to give negative results. This is bad practice and may do much harm to neurotic and suggestible patients ; besides, it puts up the cost of the hospital bed unnecessarily. In some countries it has even become fashionable to present the patient with a case sheet giving the full results of all the unnecessary investigations and analyses which have been performed. The other day I saw a healthy but rather fat school girl of sixteen who had been presented with a case sheet on which the diagnosis had been recorded as "hypopituitarism, hypothyroidism, and hypo-ovarianism" and the normal results of half a dozen blood analyses set forth. Impressive of course to an over anxious and credulous mother, but most damaging to the adolescent mind. Unnecessary investigations must be avoided and in the absence of physical signs, therefore, the provisional differential diagnoses between organic disease and anxiety neuroses, and also the decision whether to pursue investigations or not, must depend upon the history. Hence the supreme importance of the history in ordinary clinical work. The most difficult cases are those in which organic disease and anxiety neurosis occur together. Fortunately for some reason this is uncommon. When, however, it does occur, the anxiety state may mask the typical history of organic disease, but the presence of physical signs will usually save a bad mistake.

Difficulty in diagnosis also lies in minor organic states such as focal sepsis and post-influenzal debility, and slight degrees of anæmia, which may simulate the fatigue of anxiety states closely, and again in organic conditions such as gastritis which give atypical histories and no definite X-ray pictures. Hysteria is more likely to be mistaken for organic disease, particularly as it produces physical signs and the patient is not obviously "neurotic", than for anxiety neurosis, but as the hysterical symptom is produced by intervention of the mind, it seldom conforms to the same symptom when produced by organic disease. In hysterical aphonia, for instance, the patient persists in speaking in a whisper, but if asked to cough will do so loudly. Moreover, in hysteria there is a conscious or subconscious motive keeping up the symptoms, and if something can be learned of the personality, or if something be known of the patient's circumstances or environment, this may be discovered.

The underlying principles are clear, but in practice diagnosis is difficult, and mistakes are common, and have consequences for ourselves and for our patients. Some mistakes, failing to recognise a perforated duodenal ulcer for instance, may be disastrous to the patient. Other mistakes, also humiliating to our own pride and derogatory to our reputations, such as mistaking carcinoma of the stomach (for which nothing could have been done) for a simple dyspepsia matter little to the patient. But the suggestion of cancer to a patient who is really suffering from nervous dyspepsia may do the patient much harm. The consequences of mistakes of this kind are seldom realised. It is a more serious error from the patient's point of view to suggest organic disease to a neurotic person than it is to miss some organic condition for which nothing could have been done. It is therefore important to guard against suggesting organic disease to neurotic people by hesitation in diagnosis, by unguarded statements, or by unnecessary investigation. Nor is there anything particular to be gained, except in so far as it is necessary to guard ourselves, by frightening patients about conditions for which there is no particular treatment and the prognosis quite uncertain. Where high blood pressure is concerned, for instance, ignorance is bliss, and in many cases of heart disease it is foolish to make the patient wise. 
The treatment of organic disease depends upon the nature of the disease, and requires knowledge, experience, common sense, and skill, but lies outside the scope of this lecture. The treatment of "functional" cases depends just as much on accurate recognition of their cause, and although in a sense it may be less important, it is in many ways more difficult. The treatment of a primary physiological defect is to see that the particular function at fault is not over-taxed as in diabetes, to supply a physiological deficiency as in rickets or myxœedema, or to encourage the particular function at fault to develop on the right lines as in constipation. In the case of an acquired disorder of function, such as allergic asthma, steps should be taken to avoid the cause, or an attempt should be made to restore normal physiology, in this particular case, by desensitization. The treatment of anxiety neurosis is more difficult. Some perhaps would take the view that this should be left to psychologists. Whatever may be said for or against this, the fact remains that there are not enough psychologists in the country to deal with the army of neurotic people that the circumstances of modern life are creating. General practitioners must therefore deal with their own neurotic patients, the majority of whom require sympathy and discipline rather than hypnotism or psycho-analysis. It is the failure of the profession to deal with the neurotic which is largely responsible for so many patients, after consulting one specialist after another without satisfaction, or wandering from one hospital to another without relief, ultimately resorting to quacks.

The treatment of an anxiety neurosis is to eradicate fear from the patient's mind. This sounds simple enough, but while any ordinary trained medical man with a good pair of hands should be able to remove an acutely inflamed appendix without difficulty, it takes character and experience, and much more time and trouble, to eradicate from a patient's mind the fear of some disease by which he has become obsessed, and which is responsible for his symptoms.

The first thing to do in a case of anxiety neurosis, is to confirm the diagnosis, and convince the patient by a thorough physical examination that no organic disease is present. Then it is no use to tell the patient that there is nothing the matter with him for in the first place he will not believe it and in the second place it is not true. The next thing is to treat him or her as an intelligent being, and explain how symptoms can be produced by anxiety of the mind in the complete absence of organic disease of the body. This comes as a complete surprise to some patients, but many are ready to understand that mental states can affect the bodily health, and at this point it is sometimes possible to discover the particular fear which lies behind the patient's symptoms. Many patients profit enormously by just talking openly and unreservedly about their fears. If explanation fails to carry conviction, it may be necessary to resort to X-rays, for an X-ray investigation carries more conviction to the average patient than an expression of opinion based on ordinary clinical examination, and it is as legitimate to use $\mathrm{X}$-rays to convince a patient that he has nothing the matter with him as to prove the existence of some organic condition. To patients X-rays are infallible: we, however, know their limitations. Most difficulty in effecting our object will be encountered in patients with hysteria who are taking refuge behind their symptoms, while in patients with psychosis it is usually impossible.

Having thus attempted to set a patient's mind at rest by persuasion, suggestion, explanation, and perhaps X-rays, or any other investigation which may be necessary, it is essential to forbid all further self-treatment. Most anxiety neurotics have got into the habit of self-treatment and in so doing are aggravating the 
symptoms. The patient with nervous dyspepsia and a cancer phobia may diet himself to such an extent that he may become anæmic. The patient with cardiac symptoms of neurotic origin takes one patent medicine after another. The patient who has become introspective about his colon, thinking that aperients are bad for him, agonises each night over how little he can afford to take, and each morning, scared of the ill effects of constipation, worries whether he has taken enough. This does not mean that all dieting, and all aperients, and all medicine should be stopped at once, but the patient must stop treating himself and rely on his doctor for treatment. The diet should be prescribed and any anæmia treated with adequate doses of iron and not merely with a tonic. A regular night aperient should be taken and progressively reduced in quantity, if possible without the patient's knowledge. Suggestion is much more effective if at the same time you can relieve the patient's symptoms. Treat body and mind together.

In addition the patient should be warned against the danger of introspection and particularly abdominal introspection to which so many chronic dyspeptics become prone. Many neurotic people spend their spare time worrying about their health, and the less they have to do the more they worry. They have often given up all outside interests such as exercise, games, books, and hobbies. Such people must be encouraged to go back to their hobbies, to read books again, and to use their spare time for some other purpose than thinking about themselves. They should be taught that their salvation lies not in drugs and doctors but in mental hygiene, self-discipline and courage. Those who worry unreasonably over the uncertainty of human life, and unfortunately many fears are reasonable, will lean heavily upon you. Those who have lost courage, or have no faith, or no philosophy of life, will find happiness and health only in religion.

Lastly, let me ask of you two things. In the first place, to put yourself in every patient's shoes and look at the situation from every patient's point of view. This is so difficult that it requires a conscious effort on our part. Try and imagine what it is like to be a neurotic, and to have a cancer phobia. When you are relatively young, try and think what it will be like to be old, and death not far away. When you are older try and recall, when occasion requires it, what it was like to be young and inexperienced. Patients are interested in getting well, and are not or should not be interested in medicine. There is much that neurotic patients should not know, and that patients with organic disease need not be told. This does not mean that you are to be slack in your mental processes. Have two departments in your mind to which every case is referred: the one that looks at the situation from the patient's point of view, and the other which for the sake of your own intellectual honesty and intellectual development, and for the sake of the progress of medicine, looks upon every case as a problem of pure science. And that leads up to my second plea. Be as accurate as you can in diagnosis. Every diagnosis, when our knowledge permits, should consist of three parts: the pathological process from which the patient is suffering, the structural lesion which that pathological process has produced, and the disorder of function which is responsible for the patient's symptoms and which, if untreated or untreatable, may be responsible for the patient's death. "Mitral stenosis" is no diagnosis. The correct description of such a case is: chronic rheumatic carditis, the pathological process, mitral stenosis, the structural lesion, and myocardial failure, if any, the disorder of function. I have chosen a simple example, but I would particularly ask you to include in your list of pathological processes those less obvious causes of disorder of physiological function which have been the subject of my lecture to-day. 